\title{
Examination of Eggers' Relationship Between Transverse Wave Profiles and Wave Resistance
}

\author{
By H. E. Kobus²
}

\begin{abstract}
A two-dimensional vertical strut with a cross section obtained by conformal mapping of an ogive in an unbounded plane into a channel has been investigated. Eggers' relationship between transverse wave profiles and wave resistance has been applied to both analytical and experimental profiles. The basic assumption of a free wave system in the analysis seems to introduce only a small error in comparison to the effects of viscosity.
\end{abstract}

Is RECENT years, several attempts have been made to obtain the wavemaking resistance of a floating body directly from measurements of the wave pattern following it. A relationship derived from linearized potential theory by Eggers $[1,2]^{3}$ seems the most promising, since it is based on the single assumption of the presence of a system of free waves, or, in other words, that local effects have vanished in the region of measurement and the wave system has assumed the asymptotic form. The results obtained from such an analysis, however, seem to be in poor agreement with experimental values, which indicates some shortcomings in the underlying assumptions: firstly, measurements are performed at moderate dis-

1 This research was performed under partial sponsorship of the Bureau of Ships Fundamental Hydromechanics Research Program, administered by the David Taylor Model Basin, Contract Nonr $1611(0 \overline{)})$.

${ }^{2}$ Research Associate, Institute of Hydraulic Research, University of Iowa, Iowa City, Iowa.

${ }^{3}$ Numbers in brackets designate References at end of paper.

Manuscript received at SNAME Headquarters, December 16, 1966 . tances behind the body, where the influence of the "nearfield term" may not be negligible, and secondly, the presence of a viscous wake has a pronounced effect on the surface configuration, which is not accounted for in the analysis.

The purpose of this study is to examine the importance of these two simplifications in Eggers' analysis by analytical and experimental means under nearly ideal conditions. A slender, two-dimensional ogival strut is investigated. Eggers' method is applied to transverse surface profiles, obtained both from linearized potential theory and from experiments, and the results are compared with the theoretical wave resistance as obtained from the known zero-Froude-number singularity distribution generating the body.

\section{Surface Elevation Due to a Line Source in a Channel}

The velocity potential for a source of strength $m$ moving at a constant speed $U$ below the free surface of a semi-infinite fluid was first given by Havelock and since has been rederived by many. In a right-handed cartesian coordinate system, fixed with respect to the source,

\footnotetext{
$b=\operatorname{tank}$ width

$d, d^{\prime}=$ abscissae of source in conformal mapping of ogive

$g=$ gravitational acceleration

$h=$ depth of tank

$m=$ source strength

$n^{*}=\left[1+\left(\frac{4 \pi}{b \mathrm{~K}_{0}}\right)^{2} n^{2}\right]^{1 / 2}$

$u, v=$ velocity components in the $x$ - and $y$-direction

$x, y, z=$ right-handed cartesian $\mathrm{co}^{-}$ ordinates
}

$$
\begin{aligned}
& x_{i,}, y_{0},-z_{0}=\text { coordinates of point source } \\
& x_{r}=\text { real part of complex num- } \\
& \text { ber } z_{c} \\
& z, z_{1}, z_{c}, \zeta=\text { complex variables in con- } \\
& \text { formal mapping of ogive } \\
& F A(\beta)=2 \cdot \int_{0}^{L / 2} m\left(x_{0}\right) \sin \left(\beta x_{0}\right) d x_{0} \\
& L=\text { length of modified ogive } \\
& R=\text { theoretical wave resistance } \\
& C^{*}=\text { forward velocity of the } \\
& \text { source } \\
& W=\text { complex potential }
\end{aligned}
$$

$$
\begin{aligned}
Z_{0}, Z_{1} & =\frac{\mathrm{K}_{0} b}{4 \pi n} \pm\left[\left(\frac{\mathrm{K}_{0} b}{4 \pi n}\right)^{2}+1\right]^{1 / 2} \\
\alpha & =\text { opening angle of modified } \\
\gamma & =2 /(2-\alpha / \pi) \\
\zeta & =\text { surface elevation } \\
\zeta_{s}, \zeta_{1}, \zeta_{2} & =\text { components of surface ele- } \\
\xi_{n}^{*} & =\left[\xi^{2}+\left(\frac{2 \pi n}{b}\right)^{2}\right]^{1 / 3}>0 \\
\rho & =\text { mass density } \\
\mathrm{K}_{0} & =g / U^{2} \\
\phi & =\text { velocity potential }
\end{aligned}
$$


which has its $x$ - and $y$-axes in the undisturbed free surface and the $z$-axis pointing vertically upward and which moves with a constant speed $U$ in the negative $x$-direction, the potential is given by

$$
\begin{aligned}
& \phi(x, y, z)=-m\left(\frac{1}{R}+\frac{1}{R^{\prime}}\right) \\
& -\frac{4 m}{\pi} \int_{0}^{\pi / 2} \mathcal{X}_{0}^{\infty} \frac{k e^{-k\left(z_{0}-z\right)}}{\left(\mathrm{K}_{0} \sec ^{2} \theta-k\right)} \cos \left[k\left(x-x_{0}\right) \cos \theta\right]
\end{aligned}
$$

$\times \cos \left[k\left(y-y_{0}\right) \sin \theta\right] d k d \theta-4 m K_{0} \int_{0}^{\pi / 2} e^{-\kappa_{0} \sec ^{2} \theta\left(z_{0}-z\right)}$

$$
X \sin \left[\mathrm{K}_{0}\left(x-x_{0}\right) \sec \theta\right]
$$$$
\times \cos \left[\mathrm{K}_{0}\left(y-y_{0}\right) \sec \theta \tan \theta\right] \sec ^{2} \theta d \theta
$$

where $P\left(x_{0}, y_{0},-z_{0}\right)$ designates the location of the source, the symbol $f$ indicates a Cauchy principal-value integral, and

$$
\begin{aligned}
\mathrm{K}_{0} & =g / U^{2} \\
R & =\left[\left(x-x_{0}\right)^{2}+\left(y-y_{0}\right)^{2}+\left(z+z_{0}\right)^{2}\right]^{1 / 2} \\
R^{\prime} & =\left[\left(x-x_{0}\right)^{2}+\left(y-y_{0}\right)^{2}+\left(z-z_{0}\right)^{2}\right]^{1 / 2}
\end{aligned}
$$

This expression vanishes to the order $(1 / x)$ for large negative values of $x$, whereas far downstream it assumes the much less rapidly vanishing asymptotic form

$\phi \approx-S m \mathrm{~K}_{0} \int_{0}^{\pi / 2} e^{-\mathrm{K}_{0}\left(z_{0}-z\right) \sec ^{2} \theta} \sin \left[\mathrm{K}_{0}\left(x-x_{0}\right) \sec \theta\right]$

$\times \cos \left[\mathrm{K}_{0}\left(y-y_{0}\right) \sec \theta \tan \theta\right] \sec ^{2} \theta d \theta \quad$ for $x \gg 0$

The potential for a vertical line source of constant strength, extending to infinite depth, results from integrating equation (1) with respect to $z_{0}$ :

$$
\begin{aligned}
& \phi_{\text {line }}=-m\left[\lim _{L \rightarrow \infty} \int_{0}^{L}\left(\frac{1}{R}+\frac{1}{R^{\prime}}\right) d z_{0}\right] \\
& -\frac{4 m}{\pi} \int_{0}^{\pi / 2} \mathcal{X}_{0}^{\infty} \frac{e^{k z}}{\left(\mathrm{~K}_{0} \sec ^{2} \theta-k\right)} \cos \left[k\left(x-x_{0}\right) \cos \theta\right] \\
& \quad \times \cos \left[k\left(y-y_{0}\right) \sin \theta\right] d k d \theta-4 m \int_{0}^{\pi / 2} e^{\mathrm{K}_{0 z} \sec 2 \theta}
\end{aligned}
$$$$
X \sin \left[\mathrm{K}_{0}\left(x-x_{0}\right) \sec \theta\right] \cos \left[\mathrm{K}_{0}\left(y-y_{0}\right) \sec \theta \tan \theta\right] d \theta
$$

The surface elevation at any point is given by

$$
\zeta(x, y)=-\left.\frac{U}{g} \cdot \frac{\partial \phi}{\partial x}\right|_{z=0}
$$

which yields

$$
\zeta(x, y)=\zeta_{s}+\zeta_{1}+\zeta_{2}
$$

with

$$
\begin{aligned}
\zeta_{s}= & \frac{-2 U m\left(x-x_{0}\right)}{g\left[\left(x-x_{0}\right)^{2}+\left(y-y_{0}\right)^{2}\right]} \\
\zeta_{1}=- & \frac{4 U m}{\pi g} \int_{0}^{\pi / 2} \mathcal{X}_{0}^{\infty} \frac{k \cos \theta}{\left(\mathrm{K}_{0} \sec ^{2} \theta-k\right)} \\
& \quad \times \sin \left[k\left(x-x_{0}\right) \cos \theta\right] \cos \left[k\left(y-y_{0}\right) \sin \theta\right] d k d \theta \\
\zeta_{2}= & \frac{4 m}{U} \int_{0}^{\pi / 2} \cos \left[\mathrm{K}_{0}\left(x-x_{0}\right) \sec \theta\right]
\end{aligned}
$$$$
\times \cos \left[\mathrm{K}_{0}\left(y-y_{0}\right) \sec \theta \tan \theta\right] \sec \theta d \theta
$$

The surface elevation vanishes upstream from the line source to the order $(1 / x)$. Far downstream it assumes the asymptotic form

$$
\zeta(x, y) \approx 2 \zeta_{2} \text { for } x \gg 0
$$

which vanishes to the $\operatorname{order}(1 / \sqrt{ } x)[12]$.

If the line source is considered to lie on the centerline $\left(y_{0}=0\right)$ of a tank with walls at $y= \pm b / 2$, a double infinite series of images at $y_{0}= \pm n b ; n=1,2,3, \ldots$, is generated and the components of the surface elevation are given by

$$
\zeta_{s}=\frac{-2 U m}{g}\left(x-x_{0}\right) \sum_{n=-\infty}^{\infty}\left[\frac{1}{\left(x-x_{0}\right)^{2}+(y-n b)^{2}}\right]
$$

$\zeta_{1}=\lim _{N \rightarrow \infty}\left\{\frac{-4 U m}{\pi g} \int_{0}^{\pi / 2} \mathcal{X}_{0}^{\infty} \frac{k \cos \theta}{\left(\mathrm{K}_{0} \sec ^{2} \theta-k\right)}\right.$

$$
\left.\times \sin \left[k\left(x-x_{0}\right) \cos \theta\right]\left\{S_{1}\right\} d k d \theta\right\}
$$

$\zeta_{2}=\lim _{N \rightarrow \infty}\left\{\frac{4 m}{U} \int_{0}^{\pi / 2} \cos \left[K_{0}\left(x-x_{0}\right) \sec \theta\right]\left\{S_{2}\right\} \sec _{-}^{-0} \theta d \theta\right\}$

where $S_{1,2}$ is defined as

$$
S_{1,2}=\sum_{n=-N}^{N} \cos \left[A_{1,2}(y-n b)\right]
$$

with

$$
A_{1}=k \sin \theta ; \quad \dot{A}_{2}=\mathrm{K}_{0} \sec \theta \tan \theta
$$

According to a procedure proposed by Landweber [6], symmetry considerations and use of the relation

$$
\sum_{n=-N}^{N} \operatorname{Re}\left(e^{-i n \alpha}\right)=\frac{\sin \left[\left(N+\frac{1}{2}\right) \alpha\right]}{\sin (\alpha / 2)}
$$

lead to

$$
S_{1,2}=\cos \left(A_{1,2} y\right) \frac{\sin \left[\left(N+\frac{1}{2}\right) A_{1,2} b\right]}{\sin \left(A_{1,2} b / 2\right)}
$$

and hence to 
$\zeta_{1}=\lim _{N \rightarrow \infty}\left\{\frac{-2 U m}{\pi g} \int_{-\pi / 2}^{\pi / 2} \mathfrak{\aleph}_{0}^{\infty} \frac{k \cos \theta}{\left(\mathrm{K}_{0} \sec ^{2} \theta-k\right)}\right.$

$\times \sin \left[k\left(x-x_{0}\right) \cos \theta\right] \cos [k y \sin \theta]$

$$
\left.\times \frac{\sin \left[(2 N+1) \frac{k b}{2} \sin \theta\right]}{\sin \left(\frac{k b}{2} \sin \theta\right)} d k d \theta\right\}
$$

and a corresponding expression for $\zeta_{2}$.

The change of variables

$$
\xi=k \cos \theta, \quad \eta=k \sin \theta
$$

yields the following form:

$$
\begin{aligned}
\zeta_{1}=\lim _{N \rightarrow \infty}\left\{\frac{2 i U m}{\pi b g} \int_{-\infty}^{\infty} \mathcal{X}_{-\infty}^{\infty} \frac{\xi^{3} e^{i\left[\left(x-x_{0}\right) \xi+y \eta\right]}}{\left[\xi^{2}+\eta^{2}\right]\left[\mathrm{K}_{0}\left(\xi^{2}+\eta^{2}\right)^{1 / 2}-\xi^{2}\right]}\right. \\
\\
\left.\times \frac{\sin \left[(2 N+1) \frac{b \eta}{2}\right]}{\sin \left(\frac{b \eta}{2}\right)} d \xi\left(\frac{b d \eta}{2}\right)\right\}
\end{aligned}
$$

Application of the Dirichlet formula [6]

$$
\lim _{N \rightarrow \infty} \int_{-\infty}^{\infty} f(x) \frac{\sin [(2 N+1) x]}{\sin x} d x=\pi \sum_{n=-\infty}^{\infty} f(n \pi)
$$

leads to

$\xi_{1}=-\frac{2 U m}{b g} \sum_{n=-\infty}^{\infty} \mathcal{X}_{-\infty}^{\infty} \frac{\xi^{3} \sin \left[\left(x-x_{0}\right) \xi+2 \pi n \frac{y}{b}\right]}{\xi_{n}{ }^{* 2}\left(\mathrm{~K}_{0} \xi_{n}{ }^{*}-\xi^{2}\right)} d \xi$

where $\xi_{n}^{*}$ is defined as

$$
\xi_{n}^{*}=\left[\xi^{2}+\left(\frac{2 \pi n}{b}\right)^{2}\right]^{1 / 2}>0
$$

Symmetry considerations with respect to $\xi$ and $n$ finally yield

$$
\begin{gathered}
\xi_{1}=\frac{4 U m}{b g}\left\{\cos \left[\mathrm{K}_{0}\left(x-x_{0}\right)\right]\left(\operatorname{Si}\left[\mathrm{K}_{0}\left(x-x_{0}\right)\right]+\frac{\pi}{2}\right)\right. \\
\left.-\sin \left[\mathrm{K}_{0}\left(x-x_{0}\right)\right] C i\left[\mathrm{~K}_{0}\left(x-x_{0}\right)\right]\right\} \\
-\frac{8 U m}{b g} \sum_{n=1}^{\infty} \cos \left(2 \pi n \frac{y}{b}\right) \mathcal{X}_{0}^{\infty} \frac{\xi^{3} \sin \left[\left(x-x_{0}\right) \xi\right]}{\xi_{n}{ }^{* 2}\left(\mathrm{~K}_{0} \xi_{n}{ }^{*}-\xi^{2}\right)} d \xi
\end{gathered}
$$

Examination of the integral in this expression by the method of stationary phase shows that the contribution from the $n$th series term vanishes as $n$ becomes very large.

Corresponding treatment of the term $\zeta_{2}$ leads after lengthy manipulations to

$$
\begin{aligned}
\zeta_{2} & =\frac{4 \pi U m}{b g}\left\{\cos \left[\mathrm{K}_{0}\left(x-x_{0}\right)\right]+2 \sum_{n=1}^{\infty} \frac{1}{n^{*}}\right. \\
& \left.\times \cos \left[\mathrm{K}_{0}\left(x-x_{0}\right)\left(\frac{n^{*}+1}{2}\right)^{1 / 2}\right] \cos \left(2 \pi n \frac{y}{b}\right)\right\}
\end{aligned}
$$

with

$$
n^{*}=\left[1+\left(\frac{4 \pi}{b \mathrm{~K}_{0}}\right)^{2} n^{2}\right]^{1 / 2}
$$

The Cauchy principal-value integral in equation (9) has yet to be cast into a form which is suitable for numerical evaluation. The transformation

$$
z=\frac{b \xi_{n}{ }^{*}}{2 \pi n}
$$

changes this integral, which we designate by $I$, to

$I=-\mathcal{K}_{1}^{\infty} \frac{\left(z^{2}-1\right) \sin \left[2 \pi n\left(\frac{x-x_{0}}{b}\right)\left(z^{2}-1\right)^{1 / 2}\right]}{z\left(z-z_{0}\right)\left(z-z_{1}\right)} d z$ with the roots

$$
z_{0,1}=\frac{\mathrm{K}_{0} b}{4 \pi n} \pm\left[\left(\frac{\mathrm{K}_{0} b}{4 \pi n}\right)^{2}+1\right]^{1 / 2}
$$

where $z_{0}$ is the value at which the integrand is singular. For a numerical integration we have to write $I$ in two parts, of which we evaluate the first one by Simpson's rule and find an approximate solution for the second. If the upper limit of integration $(U L)$ of the first part is chosen sufficiently large as compared to unity, we can write with good approximation

$$
\begin{aligned}
& I=-\varkappa_{1}^{\tau L} \frac{\left(z^{2}-1\right) \sin \left[\left(\frac{x-x_{0}}{b}\right) 2 \pi n\left(z^{2}-1\right)^{1 / 2}\right]}{z\left(z-z_{0}\right)\left(z-z_{1}\right)} d z \\
& +S i\left[\left(\frac{x-x_{0}}{b}\right) 2 \pi n U L\right]-\frac{\pi}{2}
\end{aligned}
$$

The condition that the Cauchy principal value should occur in the first expression demands that $(U L)$ be chosen such that

$$
U L>\frac{g b}{2 \pi U^{2}}+1
$$

The numerical evaluation of the integral has to be handled carefully, in particular near the lower limit. The Cauchy principal value, on the other hand, imposes no problem. L. Landweber has shown (see Appendix 1) that integrals of the type

$$
\aleph_{a}^{b} \frac{f(z)}{\left(z-z_{0}\right)} d z
$$

can be integrated numerically by Simpson's rule if the principal value is selected to be an odd point in the summation and if the indeterminate value of the integrand at $z=z_{0}$ is replaced by $f^{\prime}\left(z_{0}\right)$. In the case of equation (13), we have 

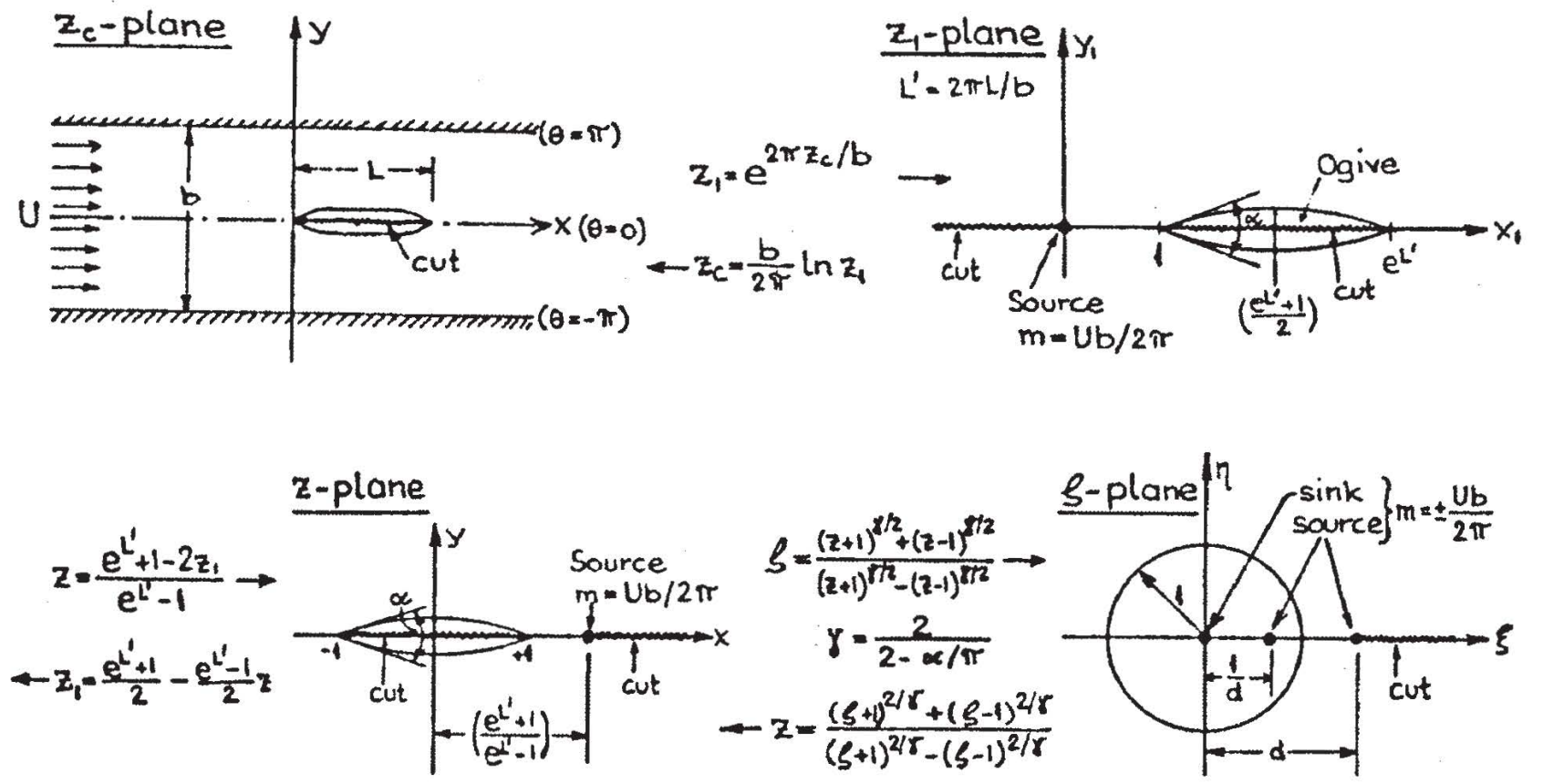

Fig. 1 Conformal mapping of flow past modified ogive in a channel into fow past unit circle

$$
\begin{array}{r}
f^{\prime}\left(z_{0}\right)=\frac{\left(2 n^{*}+1\right) \bar{a} n}{z_{0}^{2} n^{* z}} \sin \left[\left(\frac{x-x_{0}}{b}\right) 2 \pi n\left(z_{0}^{2}-1\right)^{1 / 2}\right] \\
+\frac{2 \pi \bar{a} n^{2}\left(x-x_{0}\right)}{b n^{*}}\left(z_{0}^{2}-1\right)^{1 / 2} \cos \left[\left(\frac{x-x_{0}}{b}\right)\right. \\
\left.\times 2 \pi n\left(z_{0}^{2}-1\right)^{1 / 2}\right]
\end{array}
$$

where

$$
\vec{a}=\frac{2 \pi}{b K_{0}}
$$

\section{Source Distribution for a Modiffed Ogive}

Consider a body in the $z_{c}$-plane extending from $x_{c}=0$ to $x_{c}=L$ along the centerline of a channel of width $b$, subject to a uniform stream $U$ (see Fig. 1). The transformation

$$
z=\frac{e^{2 \pi L / b}+1-2 e^{2 \pi z_{c} / b}}{e^{2 \pi L / b}-1}
$$

maps the channel region into the entire $z$-plane, and nose and stern of the body into $x= \pm 1$, respectively, whereas the uniform stream is transformed into a two-dimensional source of strength

$$
m_{s}=\frac{U b}{2 \pi}
$$

located on the positive real axis at

$$
d^{\prime}=\frac{e^{2 \pi L / b}+1}{e^{2 \times L / b}-1}
$$

Conversely, if we define the body in the $z$-plane to be an ogive of angle $\alpha$, then transformation (16) can be used to map the flow past an ogive due to an external source into flow due to a uniform stream $U$ in a channel of width $b$ past a body, which we shall call "modified ogive," located at the centerline of the channel and extending from $x_{c}=0$ to $x_{c}=L$. The problem is now reduced to finding the velocity potential for the flow past an ogive due to an external source.

The exterior of the ogive of angle $\alpha$ extending from $x=-1$ to $x=+1$ in the $z$-plane is mapped into the exterior of the unit circle by the transformation

$$
\frac{z-1}{z+1}=\left(\frac{\zeta-1}{\zeta+1}\right)^{2-\alpha / x}
$$

The source of strength $m_{4}$ on the positive real axis at $d^{\prime}$ in the $z$-plane is transformed into a source of strength $m_{s}$ on the positive real axis at a point $d$ in the $\zeta$-plane. The image system inside the unit circle due to such an external source consists of a source at the inverse point $1 / d$ and a sink at the origin, both of the same strength as the external source. The complex potential can therefore be written as

$$
W(\zeta)=m_{s}\left[\ln (\zeta-d)+\ln \left(\zeta-\frac{1}{d}\right)-\ln (\zeta)\right]
$$

and the velocities are given by 


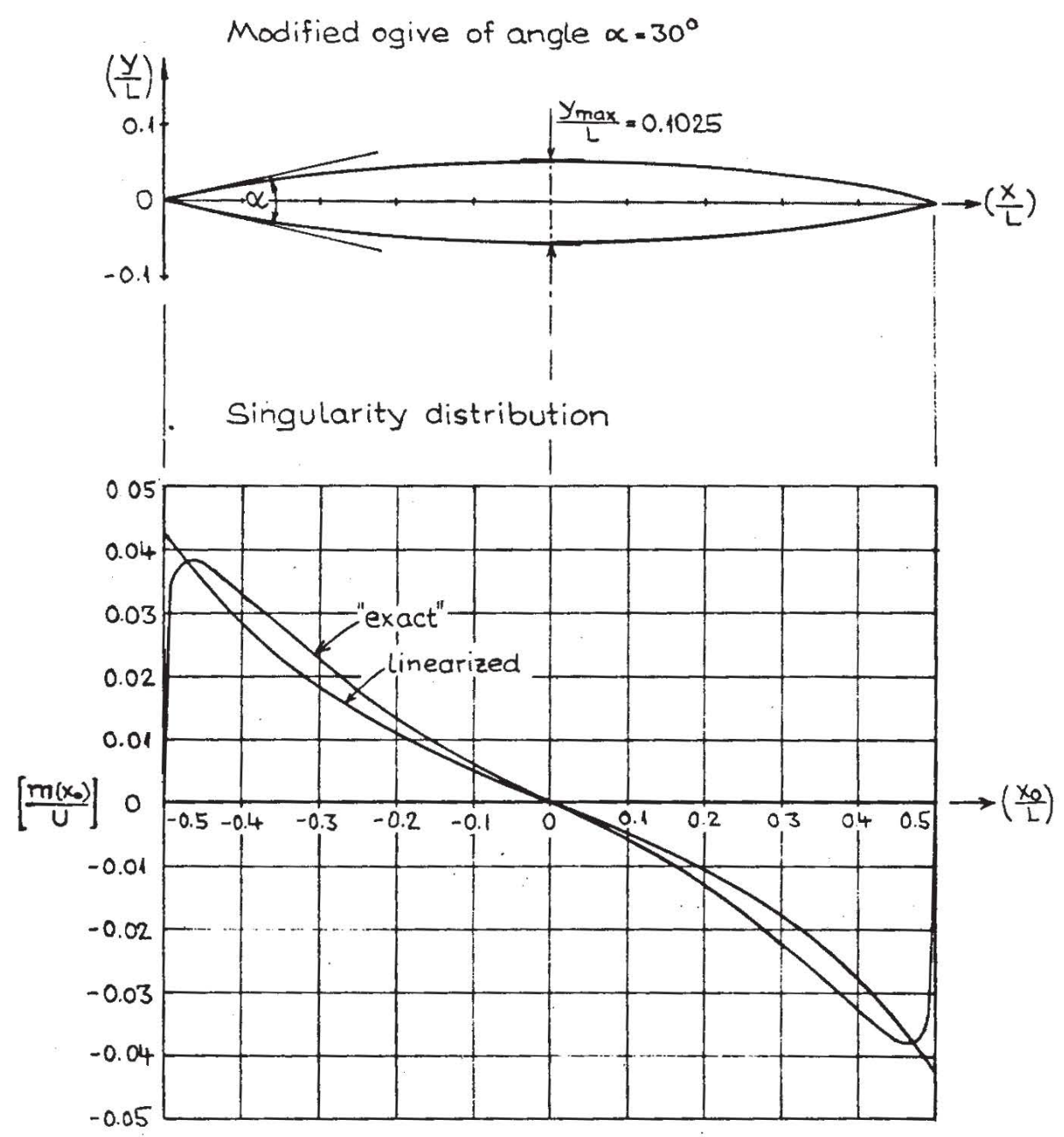

Fig. 2 Shape and singularity distribution of modified ogive

$$
\frac{d W(\zeta)}{d \zeta}=u-i v=m_{s}\left[\frac{1}{\zeta-d}+\frac{1}{\zeta-\frac{1}{d}}-\frac{1}{\zeta}\right]
$$

The relation (18) transforms this into the flow past the ogive, and the velocities in the $z$-plane are given by

$$
\frac{d W}{d z}=\frac{d W}{d \zeta} \cdot \frac{d \zeta}{d z}=\frac{d W^{(1)}}{d z}+\frac{d W^{(2)}}{d z}-\frac{d W^{(3)}}{d z}
$$

The first one of these terms is

$\frac{d W^{(1)}}{d z}=2 \gamma m_{3}\left\{\frac{\left(z^{2}-1\right)^{\gamma / 2-1}}{(1-d)(z+1)^{\gamma}+2 d(z+1)^{\gamma / 2}(z-1)^{\gamma / 2}-(1+d)(z-1)^{\gamma}}\right\}$ with

$$
\gamma=\frac{2}{2-\alpha / \pi}
$$

If $d$ is replaced by $1 / d$, then equation (20) represents the second term, and for $d=0$ it represents the third term of equation (19).

The function (19) has branch-points at $z= \pm 1$, and we have to introduce a cut along the real axis between these points in order to remain always on the same Cauchy-Riemann surface. We shall continue the flow field analytically into the body and determine the jump in normal velocity across that cut, which can then be interpreted in terms of a distribution of sources along the cut. If we define

$$
z-1=t e^{i \theta}
$$

then we have on the real axis for $x>1$ 
$\lim _{ \pm}\left(\frac{d W^{(1)}}{d z}\right)=\lim _{ \pm}\left(u_{2}{ }^{(1)}-i v_{z}{ }^{(1)}\right)=-2 \gamma m_{s}\left\{\frac{(1+x)^{\gamma / 2-1}(1-x)^{\gamma / 2-1}(\cos [\pi(\gamma / 2-1)] \pm i \sin [\pi(\gamma / 2-1)])}{a^{\prime}+b^{\prime}\left(\cos \frac{\pi \gamma}{2} \pm i \sin \frac{\pi \gamma}{2}\right)-c^{\prime}(\cos \pi \gamma \pm i \sin \pi \gamma)}\right\}$

where

$$
\begin{aligned}
a^{\prime} & =(1-d)(1+x)^{\gamma} \\
b^{\prime} & =2 d(1+x)^{\gamma / 2}(1-x)^{\gamma / 2} \\
c^{\prime} & =(1+d)(1-x)^{\gamma}
\end{aligned}
$$

$$
\begin{aligned}
m(x)=\frac{\gamma U}{2 \pi} & \sin \left(\frac{\pi \gamma}{2}\right)\left(e^{L^{\prime}}-1\right) e^{x^{\prime}} \\
& \times\left[\left(e^{L^{\prime}}-e^{x^{\prime}}\right)\left(e^{x^{\prime}}-1\right)\right]^{\gamma / 2-1} \sum_{i=1}^{3}
\end{aligned}
$$

Upon separating real and imaginary parts, we find

$$
\times \frac{\operatorname{sign}(i)(a+c)}{a^{2}+b^{2}+c^{2}+2 b(a-c) \cos \left(\frac{\pi \gamma}{2}\right)-2 a c \cos (\pi \gamma)}
$$

$$
\text { - } \Delta u_{z}^{(1)}=\lim _{+}\left(u_{z}^{(1)}\right)-\lim _{-}\left(u_{z}^{(1)}\right)=0
$$

$\Delta v_{z}^{(1)}=-4 \gamma m_{s} \sin \left(\frac{\pi \gamma}{2}\right)\left\{\frac{(1+x)^{\gamma / 2-1}(1-x)^{\gamma / 2-1}\left(a^{\prime}+c^{\prime}\right)}{a^{\prime 2}+b^{\prime 2}+c^{\prime 2}+2 b^{\prime}\left(a^{\prime}-c^{\prime}\right) \cos \left(\frac{\pi \gamma}{2}\right)-2 a^{\prime} c^{\prime} \cos (\pi \gamma)}\right\}$

This expression describes the jump in normal velocity across the cut in the $z$-plane. Since the transformation from the $z$-plane to the $z_{c}$-plane does not introduce any new branch-points, we can evaluate the discontinuity in normal velocity across the cut along the real axis in the $z_{c}$-plane immediately from the known jump in the $z$-plane and the relation

$$
\frac{d W}{d z_{c}}=\frac{d W}{d z} \cdot \frac{d z}{d z_{c}}=\frac{d W}{d z}\left[\frac{-4 \pi e^{2 \pi z_{c} / b}}{b\left(e^{2 \pi L / b}-1\right)}\right]
$$

which, together with equations (16) and (17), yields where

$$
\begin{aligned}
& a=\left(1-d_{i}\right)\left(e^{L^{\prime}}-e^{x^{\prime}}\right)^{\gamma} \\
& b=2 d_{i}\left(e^{L^{\prime}}-e^{x^{\prime}}\right)^{\gamma / 2}\left(e^{x^{\prime}}-1\right)^{\gamma / 2} \\
& c=\left(1+d_{i}\right)\left(e^{x^{\prime}}-1\right)^{\gamma}
\end{aligned}
$$

and

$$
\operatorname{sign}(i)= \begin{cases}+1 & \text { for } i=1,2 \\ -1 & \text { for } i=3\end{cases}
$$

$$
\Delta v_{z_{c}}(1)=2 \gamma U\left(e^{2 \pi L / b}-1\right) \sin \left(\frac{\pi \gamma}{2}\right)\left\{\frac{e^{2 \pi x_{c} / b}\left[\left(e^{2 \pi L / b}-e^{2 \pi x_{c} / b}\right)\left(e^{2 \pi x_{c} / b}-1\right)\right]^{\gamma / 2-1}(a+c)}{a^{2}+b^{2}+c^{2}+2 b(a-c) \cos \left(\frac{\pi \gamma}{2}\right)-2 a c \cos (\pi \gamma)}\right\}
$$

If we assume that the jump in normal velocity is generated by a distribution of three-dimensional sources, constant with respect to the third dimension and extending over an infinite width, of strength $m$ per unit length and unit width, then Gauss' flux theorem gives

$$
d Q=\left(\Delta v^{(1)}+\Delta v^{(2)}-\Delta v^{(3)}\right) d A=4 \pi m d A
$$

or

$$
m=\frac{\Delta v^{(1)}+\Delta v^{(2)}-\Delta v^{(3)}}{4 \pi}
$$

With the abbreviations

$$
x^{\prime}=2 \pi x_{c} / b ; \quad L^{\prime}=2 \pi L / b
$$

the source distribution along the centerline of the modified ogive is therefore given by with

$$
\begin{aligned}
& d_{1}=\frac{\left(e^{L^{\prime}}\right)^{\gamma / 2}+1}{\left(e^{L^{\prime}}\right)^{\gamma / 2}-1} \\
& d_{2}=\frac{\left(e^{L^{\prime}}\right)^{\gamma / 2}-1}{\left(e^{L^{\prime}}\right)^{\gamma / 2}+1} \\
& d_{3}=0
\end{aligned}
$$

The shape of the modified ogive is symmetrical with respect to the center of the body. A proof of this as given by E. O. Macagno is shown in Appendix 2.

The "linearized" three-dimensional source distribution for a body slender enough that its thickness can be assumed to be negligible is given by 

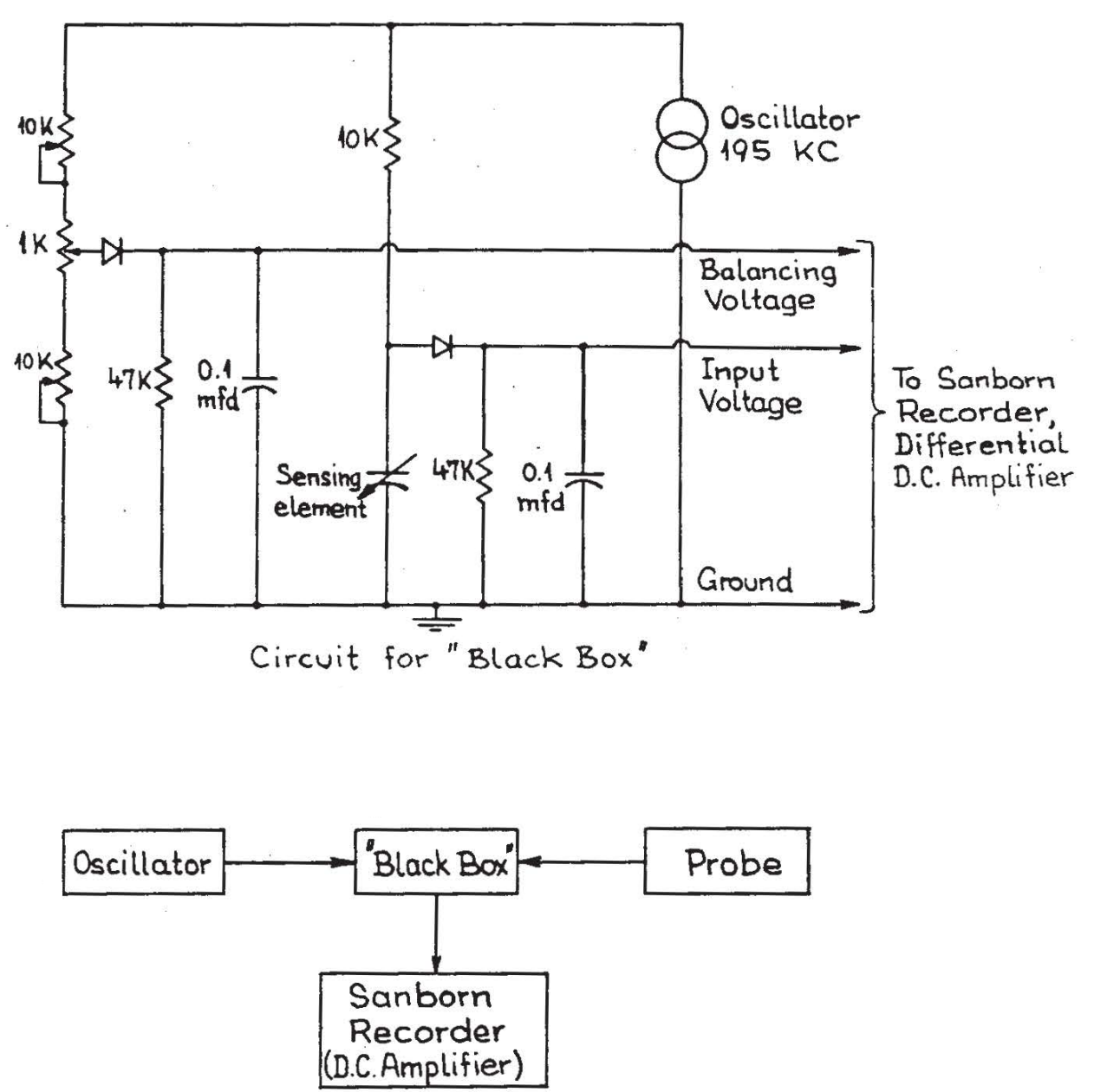

Fig. 3 Electrical circuit for capacitance probe

$$
m(x)=\frac{U}{2 \pi} \tan \theta(x)
$$

where $\tan \theta(x)$ is defined by the geometry of the modified ogive.

\section{Surface Elevation and Wave Resistance of Modified Ogive}

The surface configuration behind the modified ogive is obtained by integrating the expressions for the line source over the singularities generating the body. By shifting the coordinate system so that the origin is located at the center of the body and the modified ogive extends from $-L / 2$ to $L / 2$, by making use of the antisymmetry of the source distribution, and by defining

$$
F A(\beta)=2 \int_{0}^{L / 2} m\left(x_{0}\right) \sin \left(\beta x_{0}\right) d x_{0}
$$

we can express the surface elevation due to the modified ogive as

$$
\zeta=\zeta_{s}+\zeta_{1}+\zeta_{2}
$$

with the asymptotic form far downstream

$$
\lim _{x \rightarrow+\infty} \zeta=2 \zeta_{2}
$$

Here

$$
\begin{aligned}
\zeta_{s}=-\frac{2 U}{g} & \int_{-L / 2}^{L / 2} m\left(x_{0}\right)\left(x-x_{0}\right) \\
& \times\left[\sum_{n=-\infty}^{\infty} \frac{1}{\left(x-x_{0}\right)^{2}+(y-n b)^{2}}\right] d x_{0}
\end{aligned}
$$

and

$$
\begin{aligned}
\zeta_{1}= & \frac{4 U}{b g} \int_{-L / 2}^{L / 2} m\left(x_{0}\right)\left\{\cos \left[\mathrm{K}_{0}\left(x-x_{0}\right)\right]\right. \\
& \times\left(S i\left[\mathrm{~K}_{0}\left(x-x_{0}\right)\right]+\frac{\pi}{2}\right)-\sin \left[\mathrm{K}_{0}\left(x-x_{0}\right)\right] C i
\end{aligned}
$$$$
\begin{aligned}
& \left.\times\left[\mathrm{K}_{0}\left(x-x_{0}\right)\right]\right\} d x_{0}-\frac{8 U}{b g} \sum_{n=1}^{\infty} \cos \left(2 \pi n \frac{y}{b}\right)\left\{\mathscr{X}_{1}^{U L}\right. \\
& \times \frac{\left(z^{2}-1\right) \cos \left[2 \pi n \frac{x}{b}\left(z^{2}-1\right)\right] \cdot F A\left[\frac{2 \pi n}{b}\left(z^{2}-1\right)^{1 / 2}\right]}{z\left(z-z_{0}\right)\left(z-z_{1}\right)} d z
\end{aligned}
$$$$
\left.+\int_{-L / 2}^{L / 2} m\left(x_{0}\right) S i\left[\left(\frac{x-x_{0}}{b}\right) 2 \pi n U L\right] d x_{0}\right\}
$$ 
and

$$
\begin{gathered}
\zeta_{2}=\frac{4 \pi U}{b g}\left\{\sin \left(\mathrm{K}_{0} x\right) F A\left(\mathrm{~K}_{0}\right)+2 \sum_{n=1}^{\infty} \frac{\cos \left(2 \pi n \frac{y}{b}\right)}{n^{*}}\right. \\
\left.\times \sin \left[\mathrm{K}_{0} x\left(\frac{n^{*}+1}{2}\right)^{1 / 2}\right] F A\left[\mathrm{~K}_{0}\left(\frac{n^{*}+1}{2}\right)^{1 / 2}\right]\right\}
\end{gathered}
$$

The numerical evaluation of these expressions is very elaborate, mainly because of the double integral inside the summation in equation (31), and because the amount of calculations necessary to determine $F A(\beta)$ increases linearly with the value of $\beta$. A plot of the function $F A(\beta)$. indicates that beyond an initial region it can be replaced by an approximation of the type

$$
F A(\beta) \approx \frac{c_{1}}{\beta^{c_{3}}} \cos \left(\beta L / 2-c_{3}\right) \text { for } \beta>2 L
$$

where the coefficients $c_{1,2,3}$ depend upon the particular values of $U, b, \alpha$ and $L$. The value of $c_{3}$ is obtained from a plot of the function $F A(\beta)$ itself, and $c_{1}$ and $c_{2}$ are determined from a log-log plot of the envelope of the curve. With a proper choice of the coefficients, this approximation was found to deviate from the exact expression by less than \pm 2 percent of the peak values.

The theoretical wave resistance of a body in an inviscid fluid in irrotational motion with the boundary conditions linearized in a manner appropriate to ships with small beam-to-length ratios is given by Michell's integral [4]. Since in our case the zero-Froude-number singularity distribution is known, we use a slightly modified version of this integral given by Maruo [7], which reduces to the form

$$
R=8 \pi \rho \int_{-\pi / 2}^{\pi / 2} F A^{2}\left(\mathrm{~K}_{0} \sec \theta\right) \cos \theta d \theta
$$

This expression is valid for a body in an infinite fluid. Therefore, in order to include the effect of the channel walls, an expression for the wave resistance of the body in a channel is derived from Lagally's theorem, following a procedure proposed by L. Landweber.

Consider two line sources $m_{1}$ and $m_{2}$, located at $\left(x_{1}, 0\right)$ and $\left(x_{2}, 0\right)$, respectively. Lagally's theorem states that the force components $F_{1,2}$ acting on source $m_{1}$ due to $m_{2}$, and $F_{2,1}$, acting on source $m_{2}$ due to $m_{1}$, are

$$
F_{1,2}=-4 \pi \rho m_{1} \bar{u}_{1} ; \quad F_{2,1}=-4 \pi \rho m_{2} \bar{u}_{2}
$$

where $\bar{u}_{1}$ is the velocity at $x_{1}$ due to $m_{2}$, integrated over the $z$-direction, and $\bar{u}_{2}$ the $z$-integrated velocity at $x_{2}$ due to the source $m_{1}$. The potential due to a line source in a channel is given by

$$
\phi=\phi_{s}+\phi_{1}+\phi_{2}
$$

where the individual components are obtained from the expressions for a point source by treatment analogous to that shown previously for the surface elevation due to a line source. With

$$
\bar{u}_{1}=\left.\int_{0}^{\infty} \frac{\partial \phi^{\left(m_{2}\right)}}{\partial x} d z\right|_{(x 1,0)} ; \quad \bar{u}_{2}=\left.\int_{0}^{\infty} \frac{\partial \phi^{\left(m_{1}\right)}}{\partial x} d z\right|_{(x, 0)}
$$

it is easily shown that the coritributions $m_{1} \bar{u}_{1}$ and $m_{2} \bar{u}_{2}$ due to $\phi_{s}$ and $\phi_{1}$ are equal and opposite in sign and therefore cancel each other. The term $\phi_{2}$, however, yields

$$
\begin{aligned}
\bar{u}_{1}= & \frac{-4 \pi m_{2}}{b \mathrm{~K}_{0}}\left\{\cos \left[\mathrm{K}_{0}\left(x_{1}-x_{2}\right)\right]\right. \\
& +2 \sum_{n=1}^{\infty} \frac{2}{n^{*}\left(n^{*}+1\right)} \cos \left[\mathrm{K}_{0}\left(x_{1}-x_{2}\right)\left(\frac{n^{*}+1}{2}\right)^{1 / 2}\right\}
\end{aligned}
$$

Here $m_{1} \bar{u}_{1}$ is equal to $m_{2} \bar{u}_{2}$, and therefore $\phi_{2}$ gives a resulting force. The source $m_{1}$ also contributes to the force acting on itself, as can be seen if we set $m_{2}=m_{1}$ and $x_{2}=x_{1}$. The effect of all singularities, including $m_{1}$ itself, upon the line source $m_{1}$ is now obtained by integration of the force component $F_{1,2}$ over all the singularities $m_{2}$, and the resistance of the modified ogive results then from a second integration over all the line sources $m_{1}$ generating the body. Symmetry considerations allow us to reduce the resulting double integral to the form

$R=\frac{16 \pi^{2} \rho}{b \mathrm{~K}_{0}}\left\{F A^{2}\left(K_{0}\right)+4 \sum_{n=1}^{\infty} \frac{F A^{2}\left[\mathrm{~K}_{0}\left(\frac{n^{*}+1}{2}\right)^{1 / 2}\right]}{n^{*}\left(n^{*}+1\right)}\right\}$

This expression gives us the theoretical wave resistance as a function of the singularity distribution, which we want to compare with the wave resistance as calculated from the surface configuration. It must be pointed out that other formulas for the wave resistance in a channel have been derived by Sretenskii and by Keldysh and Sedov.

In his paper [1], Eggers derived, with the assumption that the wave pattern is composed of a system of free waves, an expression for the wave resistance in terms of surface profiles at a pair of transverse cuts. It has been pointed out by several people that his formula contains a mathematical indeterminacy, and therefore his analysis has to be amended in such a way that at least three or more transverse profiles are evaluated by the method of least squares to yield the wavemaking resistance according to Eggers' analysis.

The surface elevation at any point $(x, y)$ is given, according to Eggers, by

$$
\begin{array}{r}
\zeta(x, y)=\frac{1}{2} \sum_{n=-\infty}^{\infty}\left[\alpha_{n} \cos \left(\omega_{n} x\right)-\beta_{n} \sin \left(\omega_{n} x\right)\right] \\
\times \cos \left[u_{n}\left(\frac{b}{2}-y\right)\right]
\end{array}
$$

where

$u_{n}=\frac{n \pi}{b}$

$\varkappa_{n}=$ positive root of $\left(\kappa_{n}^{2}-u_{n}^{2}-\mathrm{K}_{0} \varkappa_{n} \tanh \left(\varkappa_{n} h\right)=0\right)$

$\omega_{n}=\left(\varkappa_{n}^{2}-u_{n}^{2}\right)^{1 / 2}>0$ 


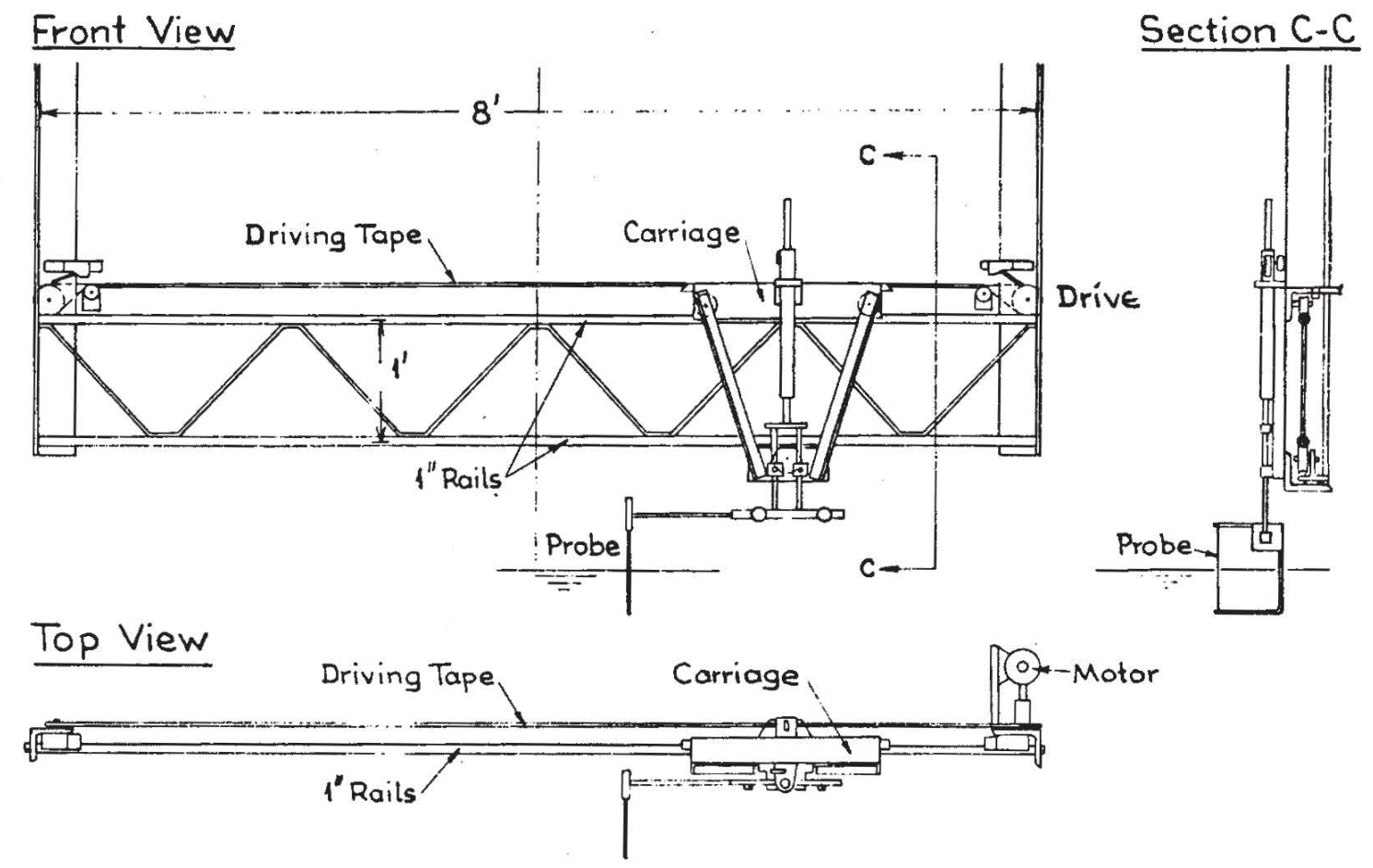

Fig. 4 Sketch of traversing mechanism

$h=$ depth of channel, in our case infinite

Multiplying both sides by $\cos \left[u_{m}\left(\frac{b}{2}-y\right)\right]$, then integrating from $-b / 2$ to $b / 2$ and making use of the orthogonality properties of trigonometric functions, one obtains

$$
\begin{aligned}
& \alpha_{n} \cos \left(\omega_{n} x\right)-\beta_{n} \sin \left(\omega_{n} x\right) \\
& \quad-\frac{2}{b} \int_{-b / 2}^{b / 2} \zeta(x, y) \cos \left[u_{n}\left(\frac{b}{2}-y\right)\right] d y=0
\end{aligned}
$$

For each $n$, the values of $\alpha_{n}$ and $\beta_{n}$ are computed from this equation by using several cuts and applying the method of least squares. Then the resistance is obtained from Eggers' equation:

$$
R=R_{0}+\sum_{n=1}^{\infty} R_{n}
$$

where

$$
R_{0}=\frac{\rho g b}{16} A_{0}\left(\alpha_{0}^{2}+\beta_{0}^{2}\right)
$$

and

$$
R_{n}=\frac{\rho g b}{8} A_{n}\left(\alpha_{n}^{2}+\beta_{n}^{2}\right)
$$

with

$$
A_{n}=2-\frac{\sinh \left(2 \varkappa_{n} h\right)+2 \varkappa_{n} h}{\cdot \sinh \left(2 \varkappa_{n} h\right)} \cdot \frac{\omega_{n}^{2}}{\varkappa_{n}^{2}}
$$

Both the calculated and the measured profiles have been evaluated according to equation (38).

\section{Experiments}

The tests were performed in the IIHR towing tank, described in [9], which is $10 \mathrm{ft}$ wide, $9 \mathrm{ft}$ deep, and $300 \mathrm{ft}$ long. A modified ogive of angle $\alpha=30 \mathrm{deg}$ with an approximate length-to-width ratio of 10:1 (Fig. 2). was tested as a vertical strut. The model is $6 \mathrm{ft}$ long and has a thickness of $0.615 \mathrm{ft}$ at the midsection. At a depth of 4 $\mathrm{ft}$ below the free surface, it is terminated by a $1 / 4$-in. aluminum plate extending $1.5 \mathrm{ft}$ to either side and $1 \mathrm{ft}$ in front of the body. Nose and stern of the model are made of solid wood, whereas the middle part consists of $1 / 4$-in. plywood sheets with horizontal $3 / 4$-in. ribs spaced 8 in. apart. All parts are coated with epoxy paint. The model was attached rigidly to the carriage, and a hole in the bottom plate, which was plugged during runs, allowed the inside of the model to fill with water, which made the model very nearly neutrally buoyant. In order to avoid any uncertainty as to the extent of the laminar boundary layer and to stimulate turbulence along the hull, a row of lucite pins (1/8-in. dia, $1 / 10$-in. height) was fitted at $5 / 8$-in. spacing 6 in. behind the bow.

As a sensing element, a capacitance-type probe was chosen, which was connected to the electrical circuit shown in Fig. 3, designed by J. Glover. A commercially available "Ceroc" wire with a ceramic-heavy teflon coating was found to be most suitable. It shows sufficient sensitivity (1/1000-ft change in surface elevation produces a signal of about $0.4 \mathrm{~mm}$ ), low surface-tension effects (about $\pm 0.0008 \mathrm{ft}$ ), and fair linearity of the output signal. The response to changes in surface elevation was found to be practically instantaneous, which is confirmed by a study of Pearlman [10]. The insulation of 

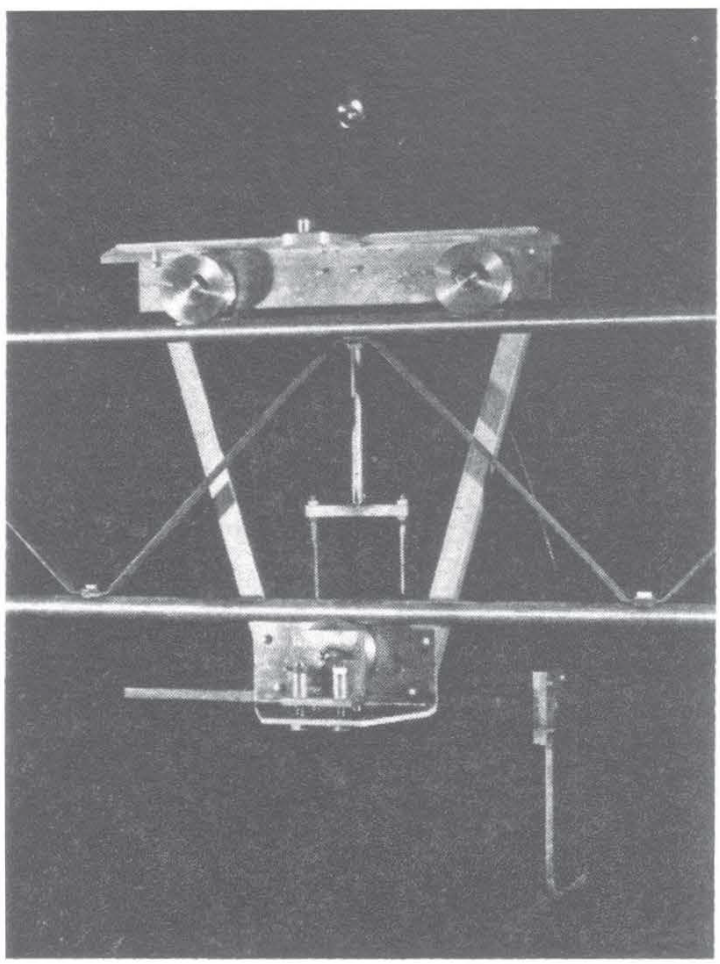

Fig. 5 Traversing mechanism

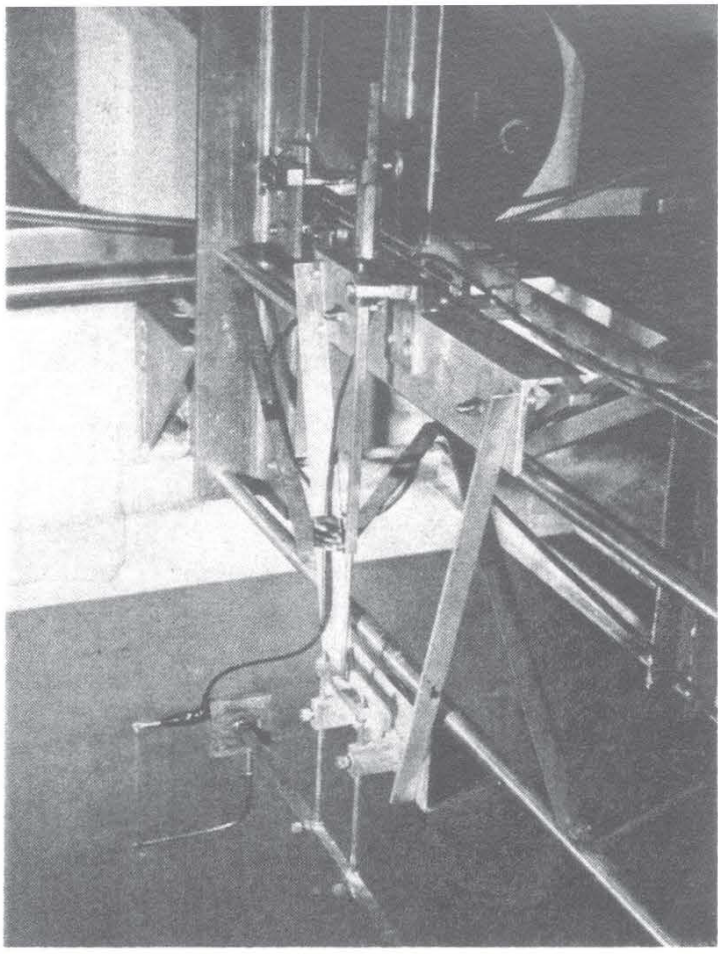

the wire slowly absorbs water, thus causing a gradual drift of the output signal. However, after the wire has been immersed for about $2 \mathrm{hr}$, the rate of drift is diminished considerably, and after $24 \mathrm{hr}$ of immersion it disappears completely. Consequently the probe is immersed at least $24 \mathrm{hr}$ before any measurements are taken, and also during the waiting periods between runs.

The traversing mechanism, which transports the probe across the channel during a run, is shown in Figs. 4 and 5. It is mounted on a trailer, which was set at distances from 3 to $25 \mathrm{ft}$ behind the stern of the model. A small carriage holding the probe is pulled along two rails from one side of the tank to the other by a constant-speed $1 / 50$-hp motor at a velocity of $0.5 \mathrm{fps}$. The motor can be switched on and off from the main carriage, and limiting switches on either end stop it automatically. A marker records every foot of travel, and thus a continuous record of probe position versus surface elevation is obtained. The probe can be mounted on either side of the probe carrier, and it can be moved both horizontally and vertically. The vertical adjustment is controlled by a vernier scale, which is used for the static calibration of the probe. A dynamic calibration is obtained by running the probe at various speeds without the ship model.

All measurements were taken at a carriage speed of 5 fps with a variation of about \pm 1 percent. At each section, two runs were performed, the probe being mounted once on either side of the probe carrier. Before each run, a null reading was obtained by taking a traverse in the quiet pool. Since the traversing mechanism yields measurements only up to 6 in. from either tank wall, a camera was mounted on the trailer and triggered automatically during the run to take a picture of an aluminum plate, fixed stationary to the tank wall, with horizontal lines $1 / 10 \mathrm{in}$. apart, from which then the surface elevation at the wall itself was obtained.

The records were read at intervals of $0.05 \mathrm{ft}$. After correcting for the null reading from the traverse in the quiet pool and for the deflection due to the velocity of travel, the readings were converted to surface elevations by means of the calibration curve. For each section the mean values from the two runs were taken in the region of overlap, and the profiles were extended to the points measured at the wall. The experimental surface profiles obtained in this manner were combined in groups of 3 consecutive sections, and the wave resistance was calculated from the first 50 terms of the infinite series in equation (38).

\section{Discussion of Results}

Transverse wave profiles across the entire tank width were measured at a Froude number of 0.36 in a region extending from $1 / 2$ model length to 4 model lengths behind the stern. The overall accuracy of the measurements is about $\pm 0.003 \mathrm{ft}$. All profiles were measured twice, and the repeatability was found to be about $\pm 0.008 \mathrm{ft}$. Close to the model the repeatability is better than far downstream, and at 4 model lengths behind the stern the profiles were found to be quite unstable. This is most likely due to imperfect reflections from the poorly aligned channel walls, in combination with the fact that at 4 model lengths the two major wave trains cross each 


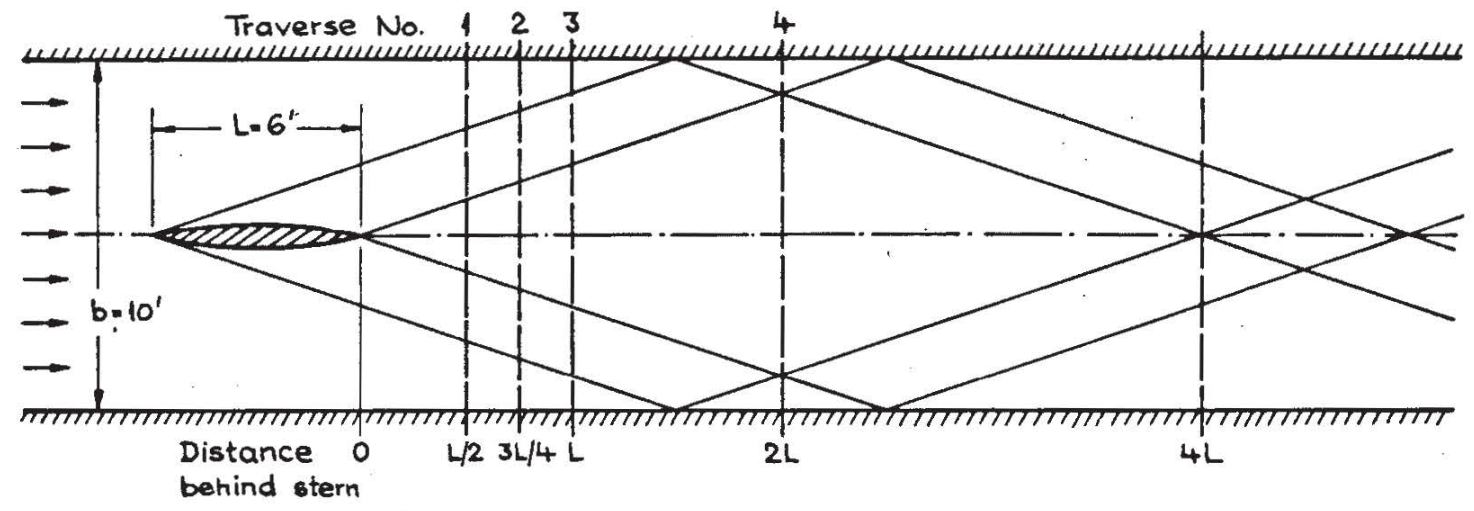

Fig. 6 Reflection pattern of bow and stern waves

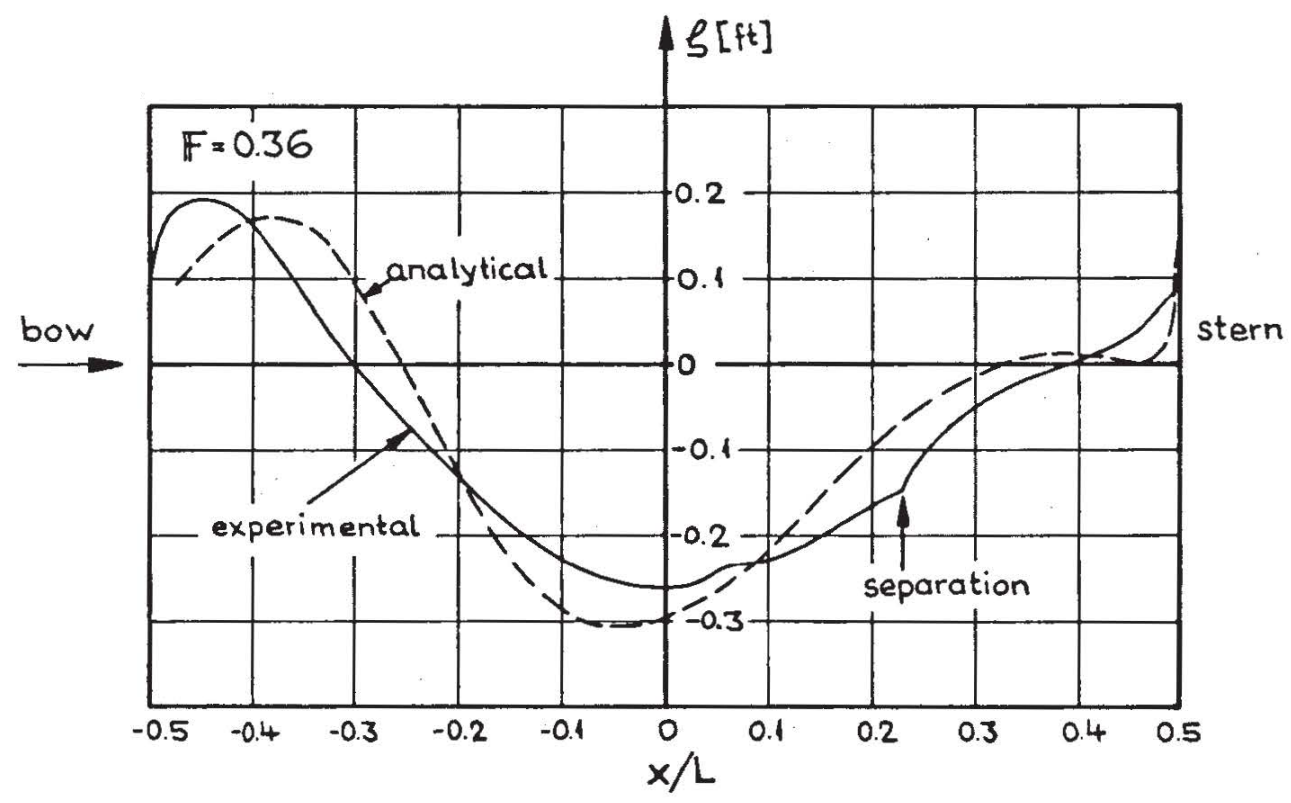

Fig. 7 Wave profile along hull

other (see Fig. 6), and therefore any inconsistency tends to be augmented at that section. The wave pattern was found to be well established and stationary with respect to the model at the time the measurements were taken; it deviates slightly from symmetry (see Figs. 8 to 11). The model alignment remained unchanged for all experiments. The wave profile along the hull of the modified ogive was measured by taking photographs of graph paper glued to the surface of the hull, and also by tracing the profile on the hull with a wax pencil. The two methods check out quite closely; the resulting profile is shown in Fig. 7. Just downstream from the midsection of the model, a small rise and subsequent drop in the water level was noticed. About halfway between midsection and stern separation occurs, which is manifested in a very rough and irregular form of the water surface downstream from the point of separation.

The profile along the hull as predicted by the analysis is shown in Fig. 7, and transverse profiles at various sections downstream from the body are given in Figs. 8 to
11. For comparison, the profiles obtained from the asymptotic expression for the surface elevation both for the "exact" and the linearized source distribution are shown on the same graphs. It is seen that for the 1:10 modified ogive the linearized singularity distribution yields wave profiles which agree quite well with those obtained from the "exact" singularity distribution. At the body itself, the asymptotic form deviates grossly from the exact form, of course, but already $1 / 2$ model length behind the stern it describes the exact form rather well and the agreement becomes better with increasing distance from the body. Both the near-field and the farfield terms are described by infinite series, which do not converge rapidly. It was found that about 50 terms of the series are needed to describe the profiles within \pm 1 percent. The computations necessary to yield the nearfield term are very much more involved than those for the far-field term, because the former contains one more integration than the latter. On the IB.I 7040 , used in this study, 200 series terms for the far-field term were 

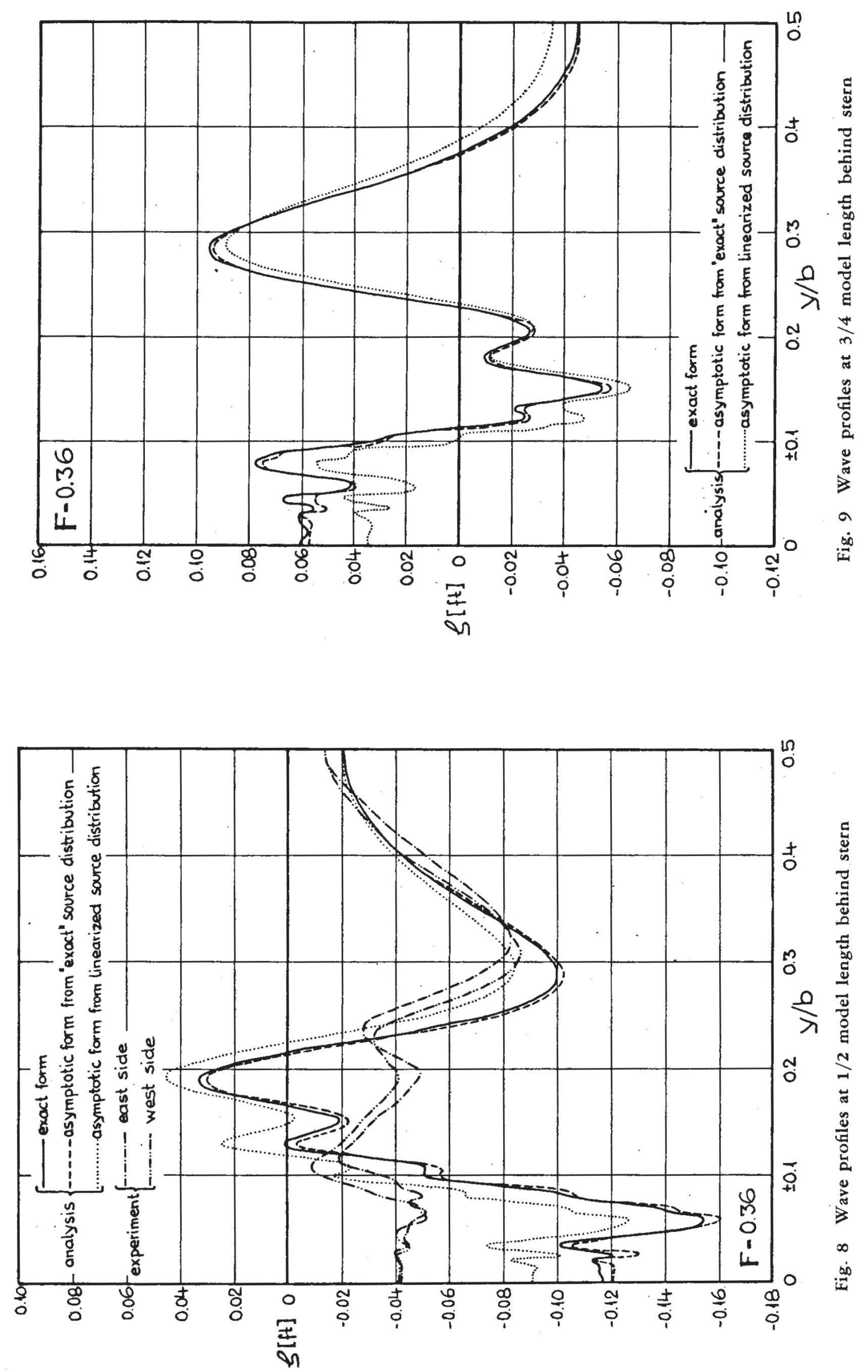
obtained in slightly more than 1 min, whereas 50 terms for the near-field term required about 45 min of computer time. The computational effort necessary to describe the near-field term increases with increasing distance from the body.

Experimental and analytical transverse profiles differ very greatly, whereas along the hull of the body they agree reasonably well. The experimental hull profile shows a higher rise in the bow region, agrees fairly well near the midsection, then shows a slower rise than the analytical profile up to the point of separation, and, oddly enough, shows fair agreement again near the stern. Similar profiles and deviations were obtained by Shearer, whose results are described in [12]. Bow and stern are the most important wave-generating regions of the body. But near the bow the experimental profile shows a significant deviation from the analytical curve, probably because of nonlinear effects, and in the stern region the flow is highly irregular and disturbed due to separation, and therefore one can hardly expect the actual waves generated by the body to resemble to any degree of accuracy the waves predicted from the analysis. At $1 / 2$ model length behind the stern, the experimental profiles exhibit the same general shape as the analytical prediction, although they differ considerably in magnitude and do not agree at all in the wake region; further downstream the agreement gets worse. In all cases, the experimental profiles display smaller amplitudes than the calculated ones. The discrepancies increase in the downstream direction due to a combination of viscous effects, particularly in the region of the wake, possibly nonlinear effects, asymmetry of the experimental profiles, which becomes increasingly important in the downstream direction, particularly after the waves have been reflected from the walls and then cross each other, and inaccuracies introduced by reflection losses and poor alignment of the channel walls. Sharma [13] also observed that theoretical and experimental wave profiles do not agree at all; in his case, the mean elevation obtained from experiments was only 56 percent of the theoretical value.

The theoretical wave resistance for the modified ogive in the channel at a Froude number of 0.36 was found to be 1 percent higher than the corresponding value for the same body in a fluid not bounded by channel walls. As a check, some asymptotic profiles have been evaluated according to Eggers' formula, and the results agreed within 1 percent with the theoretical wave resistance from Lagally's theorem. It was found, however, that the series describing the wave resistance converges quite slowly; the 30 th series term still contributes about 1 percent of the total.

Evaluation of the exact analytical profiles by Eggers' formula in groups of 3 profiles at a time yielded results which deviate from the theoretical wave resistance by about two percent. The least-square errors $\epsilon$ are of the order of 10 percent of the $c$-values, which gives an indication of the numerical accuracy of the procedure. These results seem to indicate that for sections $1 / 2$ model length and more behind the stern of the body the influence of the near-field wave on the wave resistance is small compared to the effects resulting from the neglect of viscosity.

Evaluation of the experimental profiles in groups of 3 consecutive sections at a time gives fairly consistent results: if the value at the downstream end, where the experimental results are most unreliable, is disregarded, the results fall within \pm 7 percent of the mean value. The mean wave resistance, according to Eggers' formula, found in this manner is about 57 percent of the theoretical wave resistance. The resistance values from the experimental profiles seem to exhibit a general trend to decrease in the downstream direction, which may be caused by the increasing influence of viscous action on the wave pattern. However, it is to be noted that the accuracy of the measurements decreases in the downstream direction. Simultaneous evaluation of all experimental profiles combined yields a value considerably lower than the mean from the groups of 3 sections (49 as compared to 57 percent of the theoretical wave resistance). The leastsquare errors $\epsilon$ are about 10 percent of the $c$-values, with fluctuations up to 100 percent. A previous study on a ship model gave resistance values from Eggers' analysis of 40 percent of the residual resistance of that model. The marked increase in the resistance value obtained for the modified ogive is probably due to the considerable reduction of the viscous wake and gives an indication of the importance of the latter.

Since for distances greater than 1/2 model length the error in the evaluation of the wave resistance introduced by the presence of the near-field wave is small compared to the discrepancies one is looking for, the basic assumption of the existence of a free wave system in that region seems justified. On the other hand, the large deviations of the experimental from the analytical profiles give an indication of the importance of viscous effects. Not only is the surface elevation in the viscous wake itself greatly affected, but due to the presence of the wake the entire wave pattern changes. This effect could be accounted for by the artifice of wake sources [11], which would yield a new potential function and hence a different wave pattern.

The singularity distribution used throughout the analysis is the "zero-Froude-number distribution" for the modified ogive. This does not affect in any way the comparison between the wave resistance from Lagally's theorem and Eggers' resistance value from analytical profiles, since in both cases the same source distribution has been used, and it does not matter what the shape of the corresponding body would be at any particular Froude number. On the other hand, in evaluating the experimental results the question remains as to how the shape of the body corresponding to the given singularity distribution would differ at the test Froude number from the modified ogive, or, conversely, how the singularity distribution generating the modified ogive at the test Froude number would change, and what then the theoretical wave resistance would be with which the 

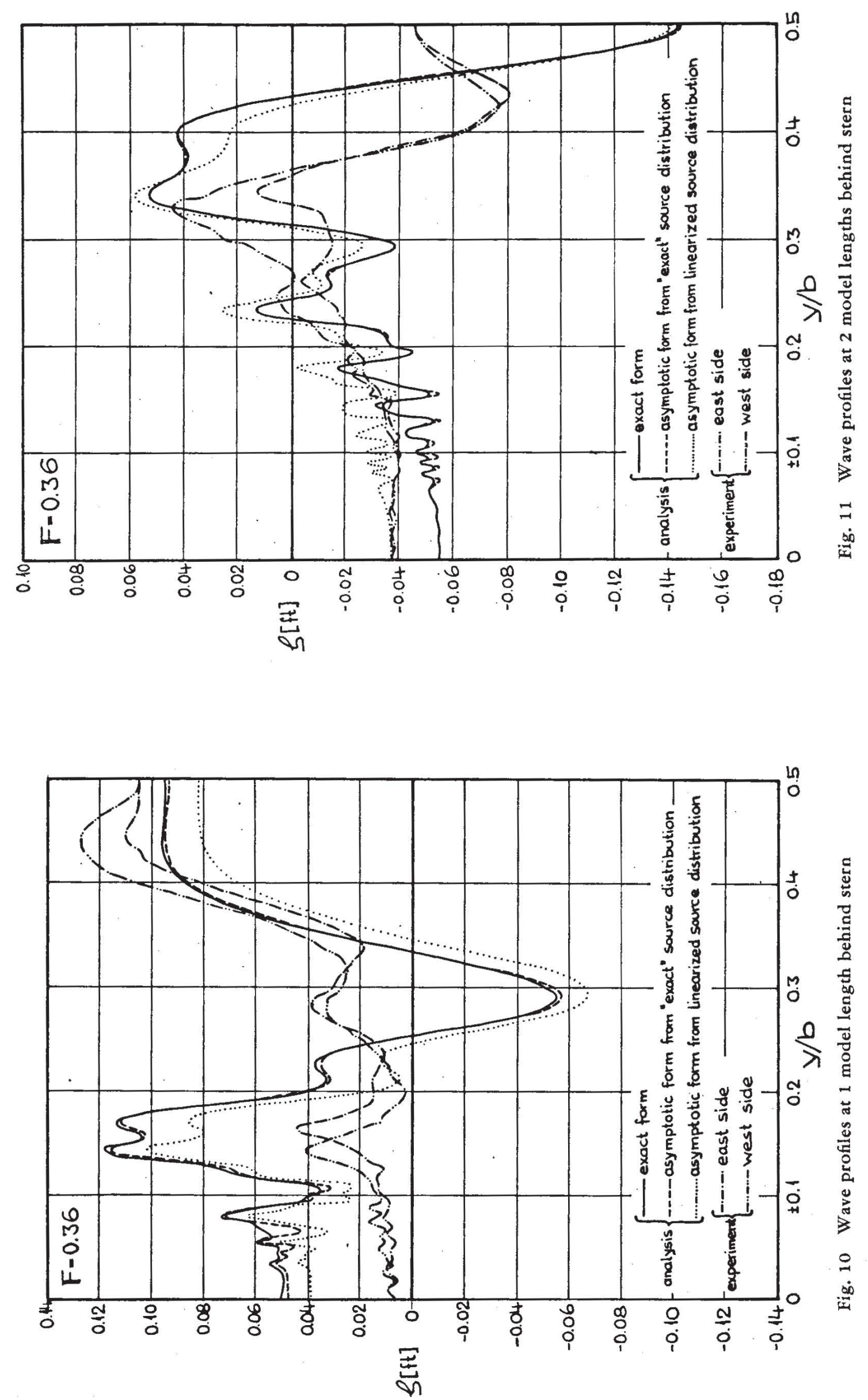


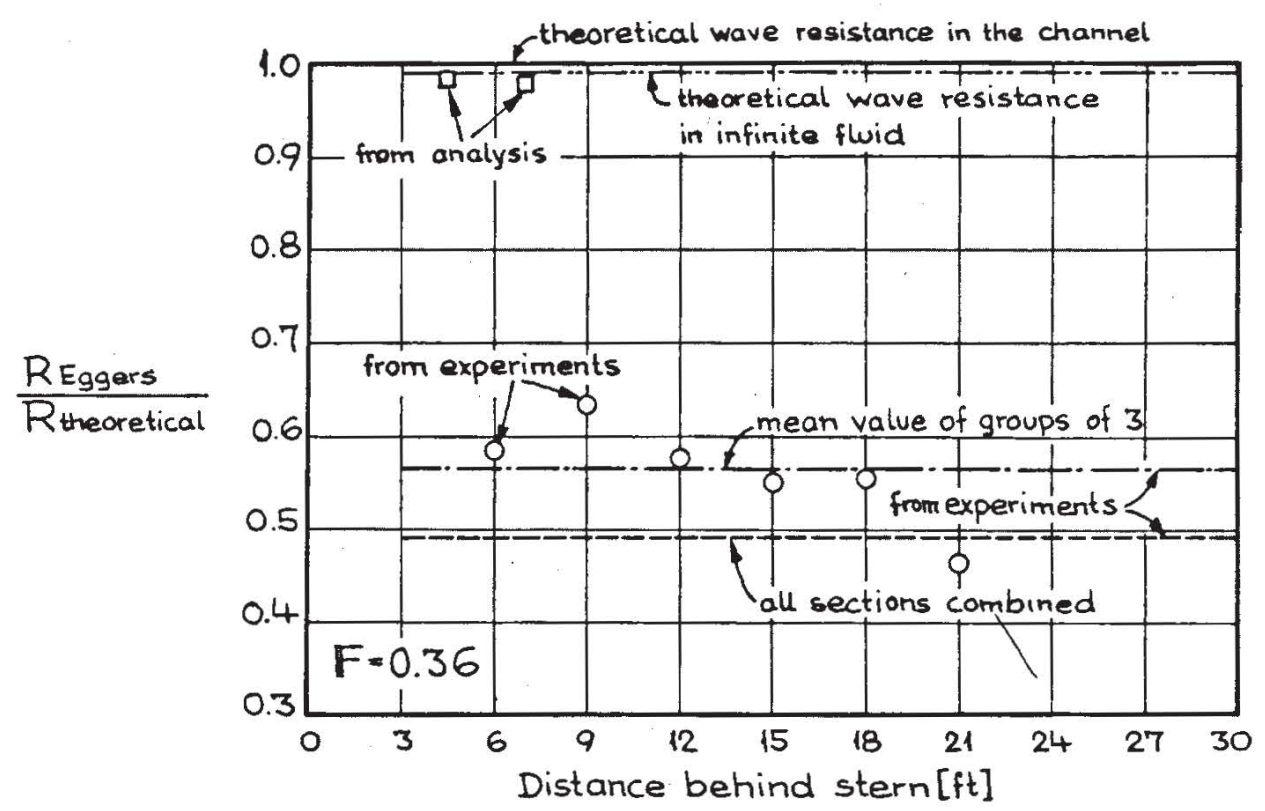

Fig. 12 Variation of resistance value from Eggers' formula with distance

experimental results ought to be compared. However, for so fine a form as was used here, one would expect the error in the theoretical wave resistance due to the use of the zero-Froude-number distribution to be small.

\section{Conclusions}

Wave profiles downstream from a body cannot be predicted in more than a qualitative manner from linearized potential theory. Whereas the profile along the hull given by the analysis matches the actual profile reasonably well, only a very general qualitative resemblance between analytical and experimental profiles is found behind the body. The agreement is better close to the body than far downstream. Theory gives generally larger amplitudes than experiment.

At 1/2 model length behind the stern, the exact analytical profile is already fairly close to its asymptotic expression for sections far downstream. At two model lengths behind the stern, the difference between the exact and the asymptotic form is negligible. The computational effort required to compute the near-field term is about 50 times as great as that for the asymptotic form. The amount of calculations for the near-field term increases with increasing distance from the body.

The asymptotic form of the wave profiles obtained from the linearized source distribution agrees fairly well with the asymptotic form from the "exact" source distribution.

The theoretical wave resistance for the modified ogive in the channel is 1 percent higher than the corresponding value for the same body in a fluid not bounded by channel walls.

For sections between $1 / 2$ and 2 model lengths behind the stern of the body, Eggers' resistance value from analytical profiles was found to differ by about two percent from the theoretical wave resistance. Therefore, by considering the order of magnitude of the discrepancies introduced by other effects, the basic assumption of a system of free waves in the analysis seems justified.

Eggers' resistance value from experimental profiles is about 57 percent of the theoretical wave resistance, which, for such a slender body, should not differ considerably from the actual wave resistance. Over the region of measurement ( $1 / 2$ model length to. 4 model lengths), a decrease in the downstream direction is noticed, which is probably due to the ever increasing influence of viscous action upon the wave pattern.

In a previous study, Eggers' resistance value for a ship model with a relatively large viscous wake was found to be 40 percent of the residual resistance. The reduction of the viscous wake therefore seems to be of great importance.

The main source of error in applying Eggers' relationship to experimental profiles seems to stem from the neglect of viscous effects in the analysis. It therefore appears to be necessary to develop a procedure of analyzing the profile measurements taking into account the presence of the viscous wake, which could possibly be accomplished by incorporating a system of wake sources [11] into the analysis.

\section{Acknowledgments}

This study was conducted at the Institute of Hydraulic Research, University of Iowa, Iowa City, Iowa, under partial sponsorship of the Bureau of Ships Fundamental 
Hydromechanics Program, Project Nonr. 1611(05), technically administered by the David Taylor Model Basin. The writer is deeply indebted to Dr. L. Landweber for the original suggestion. of this study, for his guidance and helpful advice, and for his encouragement during the course of the work, and to Mr. J. C. Tatinclaux for his careful revision of the paper. Many valuable suggestions during the design and construction of the equipment from Mr. D. Harris, Shop Supervisor, and Dr. P. G. Hubbard are gratefully acknowledged.

\section{References}

1 K. Eggers, "Über die Ermittlung des Wellenwiderstandes eines Schiffsmodells durch Analyse seines Wellensystems," Schiffstechnik, Band 9, Heft 46, 1962.

2 K. Eggers, "Über die Ermittlung des Wellenwiderstandes eines Schiffsmodells durch Analyse seines Wellensystems, Teil 2," Schiffstechnik, Band 10, Heft 52, 1963.

$3 \mathrm{~K}$. Wieghardt, "Measurements in the Field of Flow on Two Afterbody Models," David Taylor Model Basin Translation 277, 1959.

4 J. V. Wehausen, "Surface Waves," Encyclopedia of Physics, edited by S. Flügge, vol. 9, Fluid Dynamics III, Springer Verlag, Berlin, Germany, 1960.

. 5 K. Lunde, "On the Linearized Theory of Wave Resistance for Displacement Ships in Steady and Accelerated Motion," Trans. SNAME, vol. 59, 1951.

6 L. Landweber, "An Evaluation of the Method of Direct Determination of Wavemaking Resistance from Surface-Profile Measurements," Proceedings of the International Seminar on Theoretical Wave Resistance, Ann Arbor, Mich., vol. 2, August 1963.

7 H. Maruo, "Theory of Wave Resistance of Ships Moving With Constant Velocity," Stevens Institute of Technology, Note Nr. 699, 1963.

8 E. O. Tuck, "On Line Distribution of Kelvin Sources," Journal of Ship Research, vol. 8, no. 2, September 1964, pp. 45-52.

9 M. Martin, "The Iowa Towing Tank," IIHR Report to ONR, 1956.

10 M. D. Pearlman, "Dynamic Calibration of Wave Probes," MIT, 1963.

11 L. Landweber and Jin Wu, "The Determination of the Viscous Drag of Submerged and Floating Bodies by Wake Surveys," Journal of Ship Research, vol. 7, June 1963, pp. 1-6.

12 A. A. Kostyukov, "Theory of Ship Waves and Wave Resistance," State Union Publishing House for the Shipbuilding Industry, Leningrad, Russia, 1959.

13 S. D. Sharma, "Untersuchungen über den Zähigkeits- und Wellenwiderstand mit besonderer Berücksichtigung ihrer Wechselwirkung," Institut für Schiffbau der Universität Hamburg, Germany, Bericht Nr. 138, December 1964.

\section{Appendix 1}

\section{Evaluation of Cauchy Principal-Value Integrals by Quadrature Formulas}

Integrals of the type

$$
I=\mathscr{X}_{a}^{b} \frac{f(x)}{x-x_{0}} d x ; \quad a<x_{0}<b
$$

can be evaluated numerically by quadrature formulas, if the indeterminate value of the integrand at $x=x_{0}$ is replaced by $f^{\prime}\left(x_{0}\right)$. The following proof has been derived by L. Landweber. The change of variables

$$
z=2 x_{0}-x ; \quad g(z)=f(x)
$$

for the second part of the expression

$$
I=\lim _{\epsilon \rightarrow 0}\left(\int_{a}^{x_{0}-\epsilon} \frac{f(x)}{x-x_{0}} d x+\int_{x_{0}+\epsilon}^{b} \frac{f(x)}{x-x_{0}} d x\right)
$$

gives

$$
I=\lim _{\epsilon \rightarrow 0}\left(\int_{a}^{x_{0}-\epsilon} \frac{f(x)}{x-x_{0}} d x-\int_{2 x_{0}-b}^{x_{0}-\epsilon} \frac{g(z)}{z-x_{0}} d z\right)
$$

In terms of a quadrature formula-for instance, Simpson's rule- -this becomes

$$
\begin{aligned}
I=\frac{\Delta h}{3} & {\left[\frac{f(a)}{a-x_{0}}+4 \frac{f(a+\Delta h)}{a+\Delta h-x_{0}}+2 \frac{f(a+2 \Delta h)}{a+2 \Delta h-x_{0}}\right.} \\
& \left.+\ldots+4 \frac{f\left(x_{0}-\Delta h\right)}{-\Delta h}+\lim _{\epsilon \rightarrow 0}\left(\frac{f\left(x_{0}-\epsilon\right)}{-\epsilon}\right)\right] \\
& -\frac{\Delta h}{3}\left[\frac{g\left(2 x_{0}-b\right)}{x_{0}-b}+4 \frac{g\left(2 x_{0}-b+\Delta h\right)}{x_{0}-b+\Delta h}\right. \\
+ & 2 \frac{g\left(2 x_{0}-b+2 \Delta h\right)}{x_{0}-b+2 \Delta h}+\ldots+4 \frac{g\left(x_{0}-\Delta h\right)}{-\Delta h} \\
& \left.+\lim _{\epsilon \rightarrow 0}\left(\frac{g\left(x_{0}-\epsilon\right)}{-\epsilon}\right)\right]
\end{aligned}
$$

or, after reversing the transformation for the second part,

$$
\begin{aligned}
I=\frac{\Delta h}{3} & {\left[\frac{f(a)}{x-x_{0}}+4 \frac{f(a+\Delta h)}{a+\Delta h-x_{0}}+2 \frac{f(a+2 \Delta h)}{a+2 \Delta h-x_{0}}\right.} \\
& +\ldots+4 \frac{f\left(x_{0}-\Delta h\right)}{-\Delta h}-\lim _{\epsilon \rightarrow 0}\left(\frac{f\left(x_{0}-\epsilon\right)}{\epsilon}\right) \\
& +\lim _{\epsilon \rightarrow 0}\left(\frac{f\left(x_{0}+\epsilon\right)}{\epsilon}\right)+4 \frac{f\left(x_{0}+\Delta h\right)}{\Delta h}+\ldots \\
& \left.+2 \frac{f(b-2 \Delta h)}{b-2 \Delta h-x_{0}}+4 \frac{f(b-\Delta h)}{b-\Delta h-x_{0}}+\frac{f(b)}{b-x_{0}}\right]
\end{aligned}
$$


From the definition

$$
f^{\prime}\left(x_{0}\right)=\left.\frac{d f(x)}{d x}\right|_{x=x_{0}}=\lim _{\epsilon \rightarrow 0}\left[\frac{f\left(x_{0}+\epsilon\right)-f\left(x_{0}-\epsilon\right)}{2 \epsilon}\right]
$$

it follows therefore that

$$
\begin{array}{r}
I= \\
+\ldots h+4 \frac{f\left(x_{0}+\Delta h\right)}{-\Delta h}+2 f^{\prime}\left(x_{0}\right)+4 \frac{f\left(x_{0}+\Delta h\right)}{\Delta h} \\
+\ldots+2 \frac{f(b-2 \Delta h)}{b-2 \Delta h-x_{0}}+4 \frac{f(b-\Delta h)}{b-\Delta h-x_{0}} \\
\left.+\frac{f(b)}{b-x_{0}}\right]
\end{array}
$$

This shows that a numerical integration of $I$ by quadrature formulas can proceed right through the Cauchy principal value, if the principal value itself is selected to be a discrete point of the integration and if the integrand there is replaced by the derivative of the function $f(x)$ at that point.

\section{Appendix 2}

\section{Proof of Symmetry of Modifled Ogive}

The following proof that the shape of the modified ogive is symmetrical with respect to the center of the body has been given by E. O. Macagno.

Consider the exponential transformation of the ogive in the unbounded $z_{1}$-plane into the modified ogive in the channel in the $z_{c}=$ plane (Fig. 1). Lines of constant angle $\theta$ map into horizontal lines, and lines of constant radius map into vertical lines to a logarithmic scale. Consider the tangent from the origin to the ogive in the $z_{1}$-plane $\left(\theta=\theta_{\max }\right)$, and let the distance of the tangential point from the origin be $a$. For an arbitrary radial line of angle $\theta$, designate the distance of the points of intersection from the origin by $r_{1}$ and $r_{2}$, respectively. From the geometric properties of a circle it is known that

$$
a^{2}=r_{1} r_{2}
$$

and therefore

$$
\ln a-\ln r_{1}=\ln r_{2}-\ln a
$$

which shows that the ogive will map into a symmetrical body by the exponential transformation. Conversely it is seen that only circles or circular sections are transformed into symmetrical shapes by this transformation. 